\title{
ANÁLISE DE ENVOLTÓRIA DE DADOS NO ESTUDO DA EFICIÊNCIA E DOS BENCHMARKS PARA COMPANHIAS AÉREAS BRASILEIRAS
}

\author{
João Carlos Correia Baptista Soares de Mello \\ Universidade Federal Fluminense - Niterói - RJ \\ gmajcsm@vm.uff.br
}

Lidia Angulo Meza

Universidade Veiga de Almeida - Rio de Janeiro - RJ

lidia_a_meza@yahoo.com

Eliane Gonçalves Gomes *

Embrapa Monitoramento por Satélite - Campinas - SP

eliane@,cnpm.embrapa.br

Bruno Pessôa Serapião

INSEAD - Fontainebleau Cedex - France

bruno.serapiao@alumni.insead.edu

Marcos Pereira Estellita Lins

Universidade Federal do Rio de Janeiro - RJ

lins@pep.ufrj.br

* Corresponding author/autor para quem as correspondências devem ser encaminhadas

Recebido em 11/2002, aceito em 03/2003

\section{Resumo}

Este artigo tem como objetivo avaliar a eficiência das companhias aéreas brasileiras, através do uso de Análise de Envoltória de Dados (DEA). Essa ferramenta determina a eficiência relativa de cada unidade em análise, comparando-a com as demais e considerando a relação entre insumos (inputs) e produtos (outputs). O modelo DEA usado leva em conta os retornos de escala, através da comparação de cada companhia com as que operam em escala semelhante. São considerados os dados relativos ao transporte aéreo de passageiros nos anos de 1998, 1999 e 2000, e cada companhia é considerada como uma unidade diferente em cada um dos três anos. São calculados, para cada DMU, o seu índice de eficiência e seus alvos, estes segundo o enfoque DEA-Multiobjetivo, que permite a escolha de uma meta dentre um conjunto possível de empresas eficientes.

Palavras-chave: DEA; multiobjetivo; alvos; companhias aéreas.

\begin{abstract}
This paper aims to evaluate the efficiency of Brazilian air carriers. Data Envelopment Analysis (DEA) approach is used to determine the efficiency of each airline company, comparing its outputs with available inputs. Scale of operation is taken into account. The aim is to measure each company's performance, comparing its relative efficiency to all others included in the group under investigation. It is possible to evaluate each carrier efficiency over the years (1998, 1999 and 2000), assuming that the same company is a different one in different years. Both efficiency index and targets choice are performed. The proposed approach, that uses a Multiobjective-DEA problem, allows choosing targets belonging to the feasible set of efficient companies.
\end{abstract}

Keywords: DEA; multiobjective; targets; airlines evaluation. 


\section{Introdução}

A indústria brasileira de transporte aéreo vem passando por grandes mudanças estruturais desde a década de 90. A eliminação das barreiras à entrada de novas companhias e a desregulamentação iniciada no início da década e que teve um novo impulso em 1997 (Coelho, 2002) criaram uma nova perspectiva de competição para as empresas existentes, obrigando-as a uma grande mudança em sua forma de posicionamento nesse tipo de mercado (Serapião, 2001). O mercado competitivo induziu que a gestão das empresas passasse de uma visão puramente operacional para uma visão de negócios. Nessa nova visão definem-se as rotas com base na demanda por vôos, e só então verifica-se o impacto operacional dessa definição, de forma a definir-se a malha efetiva da empresa. A grande desvalorização cambial ocorrida em 1999 revelou a ineficiência de algumas companhias em operar em mercado livre, haja vista os resultados financeiros apresentados naquela ocasião.

Até o início da desregulamentação, o mercado aéreo brasileiro era claramente dividido em companhias internacionais, nacionais e regionais, estas com área geográfica de atuação bem definida, limitando muito a concorrência entre elas. Atualmente, foram eliminadas várias das barreiras à concorrência e a divisão desses grupos não é bem definida.

Nos anos de 1998, 1999 e 2000 (anos abordados neste artigo), as companhias TAM, Transbrasil (na época com uma frota de Boeings 737 e 767), VARIG (MD11, Boeings 767, 737 e, mais recentemente, 777) e VASP (A300, Boeings 737-200, e apenas um 737-300) eram consideradas as grandes companhias nacionais e que operavam, igualmente, em rotas internacionais. Devido a sucessivas crises, a VASP deixou de operar vôos internacionais e, mais recentemente, a Transbrasil suspendeu todas as suas operações.

Entre as companhias regionais podem-se citar:

- Rio Sul (Boeing 737, Embraer 145 e 120) e Nordeste (Boeing 737, F50, Embraer 120) de grande porte, pertencentes ao Grupo VARIG;

- Interbrasil (Embraer 120) pertencente ao Grupo Transbrasil, Pantanal (ATR42) e Rico (Embraer 110 e 120, Cesna Caravan Anfíbio), dentre as de médio porte;

- TAF (Embraer 110, Cesna Caravan, Boeing 737 cargueiro) e Passaredo, de micro e pequeno porte, e que são de especial interesse deste artigo.

A companhia Passaredo era de média escala, operando com aviões Wide-Body Airbus A310 e reduziu drasticamente sua escala de operação passando a contar apenas com aviões EMB120 Brasília. Foi um movimento inverso ao da companhia TAM, que era na verdade um conglomerado de várias regionais, que operavam essencialmente aviões Cesna Caravan, F50 e F100, e que atualmente é uma companhia de grande porte, operando aviões F100, A319, A320 e A330.

Algumas companhias conhecidas não foram incluídas neste estudo. Umas, embora já operassem nos anos considerados, não são consideradas regulares pela entidade aeronáutica (DAC), muito embora realizem vôos com quase todas as características de vôos regulares. A principal dessas companhias é a Fly Linhas Aéreas, que opera no conceito de baixas tarifas, com uma frota de Boeings 727-200. A exclusão dessas companhias deve-se à ausência de dados que permitam fazer comparações com as demais, e que decorre da 
inexistência de uma legislação que obrigue à transparência de dados no setor aéreo brasileiro (Espírito Santo Jr., 2002).

Outras companhias surgiram após o período considerado neste estudo. A principal é a GOL, surgida em janeiro de 2001, com o forte conceito de low-fare e que está em rápido crescimento. Opera uma frota composta principalmente de Boeings 737-700 embora, recentemente, tenha incorporado alguns Boeings 737-800.

$\mathrm{Na}$ nova conjuntura de mercado extremamente competitivo, torna-se necessário buscar simultaneamente a eficiência operacional e um bom planejamento financeiro, visto que ambos mostram-se responsáveis pela maioria dos custos de operação desse mercado de grandes receitas, mas baixa rentabilidade. A avaliação dos aspectos não financeiros demonstra-se de grande importância, já que pode balizar mudanças que conduzam ao aumento de eficiência, em especial no que se refere ao transporte de passageiros.

Este artigo concentra-se principalmente no estudo das operações com passageiros, pois o setor de carga é pouco importante no Brasil (Burmann, 2000; DAC, 2001), devido a práticas obsoletas (Serapião, 2001). Apenas recentemente as companhias aéreas começaram a dar importância a esse segmento, sendo o transporte rodoviário de cargas o predominante no país.

Fazendo uso de Análise de Envoltória de Dados, este artigo apresenta uma abordagem alternativa para avaliar as eficiências operacional, de vendas e global de cada companhia aérea, e expande os resultados de Gomes et al. (2001) e Angulo Meza et al. (2002). Além disso, permite analisar a evolução do mercado após o início da desregulamentação e o impacto da crise de 1999.

Inicialmente descreve-se o modelo DEA usado, DEA VRS (Variable Returns to Scale), apresentando-se, em seguida, o estudo de caso e os resultados do cálculo das eficiências, feitos com o uso do software Frontier Analyst. Uma descrição detalhada do funcionamento desse software encontra-se em Angulo Meza \& Lins (2000).

O modelo para determinação de alvos alternativos é em seguida apresentado. Esse modelo é baseado em uma abordagem multiobjetivo para DEA (Angulo Meza, 2002; Angulo Meza \& Lins, 2002) usando para os cálculos o software ADBASE (Steuer, 1983). Os resultados e as conclusões obtidas são apresentados na seqüência.

Foram utilizados os dados dos Anuários Estatísticos do Departamento de Aviação Civil Brasileiro (DAC), para os anos de 1998, 1999 e 2000.

\section{Modelo DEA VRS}

A abordagem por Análise de Envoltória de Dados (Data Envelopment Analysis - DEA) foi desenvolvida por Charnes et al. (1978) para determinar a eficiência de unidades produtivas, onde não seja relevante ou não se deseja considerar somente o aspecto financeiro. No presente caso, DEA permite avaliar a eficiência relativa de cada companhia aérea (DMU Decision Making Unit) considerando-se os recursos de que dispõe (inputs) e os resultados alcançados (outputs).

O objetivo de DEA consiste em comparar um certo número de DMUs que realizam tarefas similares e se diferenciam nas quantidades de inputs que consomem e de outputs que produzem. Há dois modelos DEA clássicos: o modelo CRS ou CCR (Charnes et al., 1978),

Pesquisa Operacional, v.23, n.2, p.325-345, Maio a Agosto de 2003 
que considera retornos de escala constantes, e o modelo VRS ou BCC (Banker et al., 1984), que considera retornos variáveis de escala e não assume proporcionalidade entre inputs e outputs. Adotou-se o modelo VRS com orientação a inputs, já que interessa verificar até quanto poder-se-ia diminuir os recursos de cada companhia aérea, mantendo-se os seus níveis de operação e/ou vendas. A formulação do modelo VRS usa para cada DMU o problema de programação linear (PPL) apresentado em (1) (Lins \& Angulo Meza, 2000).

$$
\begin{aligned}
& \max h_{o}=\sum_{j=1}^{s} u_{j} y_{j o}-u_{*} \\
& \text { sujeito a } \\
& \sum_{i=1}^{m} v_{i} x_{i o}=1 \\
& \sum_{j=1}^{s} u_{j} y_{j k}-\sum_{i=1}^{n} v_{i} x_{i k} \leq 0, \quad k=1, \ldots, n \\
& u_{j}, v_{i} \geq 0 \quad \forall x, y \\
& u_{*} \in \Re
\end{aligned}
$$

Nesse modelo, para a DMU o em análise, a eficiência é dada por $h_{o} ; x_{i k}$ representa o input $i$ da DMU $k ; y_{j k}$ representa o output $j$ da DMU $k ; v_{i}$ e $u_{j}$ representam os pesos dados aos inputs $i$ e aos outputs $j$, respectivamente; $u_{*}$ é um fator de escala (quando positivo, indica que a DMU está em região de retornos decrescentes de escala; se negativo, os retornos de escala são crescentes). Se $h_{o}$ é igual a 1, a DMU $o$ em análise é considerada eficiente. As variáveis de decisão do PPL (1) são $v_{i}, u_{j}$ e $u_{*}$. De forma não matemática, no modelo VRS uma DMU é eficiente se, na escala em que opera, é a que melhor aproveita os inputs de que dispõe.

A escolha do modelo VRS vem do fato de se poder lidar com eficiências de escala, ou seja, uma companhia eficiente não precisa ter a máxima relação entre outputs e inputs, uma vez que se considera a escala de operação. Essa característica permite que companhias de tamanhos diferentes, como por exemplo, uma companhia que opera em escala nacional e internacional, tendo na sua frota grande quantidade de aviões como MD11, Boeing 767 e 737 (VARIG), e companhias que operam em rotas regionais de pequeno alcance, utilizando essencialmente uma pequena frota de aviões turbo-hélice de até 30 lugares (TRIP), sejam analisadas utilizando o mesmo modelo.

A estrutura matemática dos modelos DEA faz com que, freqüentemente, uma DMU seja considerada eficiente por serem atribuídos pesos nulos a algumas variáveis. Essas variáveis são desconsideradas na avaliação da eficiência daquela unidade, podendo acarretar uma avaliação incompleta. Torna-se possível complementar o modelo matemático adicionando restrições que permitem variar os pesos em certas faixas pré-definidas, minimizando a quantidade de variáveis que recebem peso zero (Allen et al., 1997).

Além de identificar as DMUs eficientes, os modelos DEA permitem medir e localizar a ineficiência e estimar uma função de produção linear por partes, que fornece o benchmark para as DMUs ineficientes. Esse benchmark é determinado pela projeção das DMUs ineficientes na fronteira de eficiência. Classicamente, a forma como é feita esta projeção determina orientação do modelo: orientação a inputs (quando se deseja minimizar os inputs, mantendo os valores dos outputs constantes) e orientação a outputs (quando se deseja maximizar os 
resultados sem diminuir os recursos). Existem abordagens alternativas, que podem alterar simultaneamente as quantidades de inputs e outputs. Uma destas abordagens, baseada em Programação Linear Multiobjetivo (Angulo Meza \& Lins, 2002), é usada neste artigo.

\section{Análise de Eficiência de Companhias Aéreas Brasileiras}

Uma revisão das técnicas mais avançadas de avaliação de desempenho no setor aéreo, através da utilização de avaliação de bens intangíveis, é encontrada em Serapião (2001). Estudos de eficiência que utilizam DEA e que consideram aspectos financeiros são encontrados em Fernandes \& Capobianco (2000). Um modelo com aspectos financeiros e não financeiros é usado por Adler \& Golany (2001). Neste artigo adota-se uma abordagem não financeira, com o uso de três modelos: operacional, de vendas e global. Outras abordagens não financeiras, sejam para avaliação de desempenho de companhias aéreas ou aeroportos, são encontradas em, por exemplo, Charnes et al. (1996) e Fernandes \& Pacheco (2002). Essas abordagens têm em comum o fato de calcularem uma única eficiência para cada DMU. Neste artigo são calculados três índices de eficiência, correspondentes a modelos que analisam diferentes aspetos da operação das DMUs.

Foram considerados os dados das companhias aéreas para os anos de 1998, 1999 e 2000, retirados dos Anuários Estatísticos do DAC (DAC, 1999; DAC, 2000; DAC, 2001). As DMUs do modelo consistem das companhias áreas regulares, isoladamente e agregadas em seus respectivos grupos econômicos, considerando-se que a mesma companhia em anos diferentes é uma DMU diferente (Charnes et al., 1995). Essa abordagem justifica-se por possibilitar a análise da evolução temporal de cada companhia, o que permite verificar como cada uma atravessou a grande crise de 1999, considerando que a tecnologia usada não mudou ao longo dos anos em questão. Essa é uma hipótese razoável já que, embora as variáveis ambientais tenham mudado, as frotas e técnicas de gestão não apresentaram mudanças substanciais. Caso o objetivo fosse verificar as mudanças de tecnologia e como cada DMU se comporta em relação a elas, seria necessário usar o índice de Malmquist (Färe \& Grosskopf, 1992).

O modelo que considera o aspecto meramente operacional $(M I)$ avalia, de forma rudimentar, a capacidade da empresa em colocar aviões no ar com os recursos de que dispunha. Os inputs desse modelo são quantidade de pessoal de vôo, combustível usado e a soma das capacidades de passageiros do total das aeronaves da frota; o output é dado em passageiro.km oferecidos. Uma empresa que tenha baixa eficiência neste modelo demonstra que tem possibilidade de melhorar a utilização de seus aviões ou diminuir os recursos que utiliza.

O modelo de vendas (M2) busca medir a capacidade de cada companhia em ocupar os aviões que colocou no ar. Como inputs foram utilizados quantidade de pessoal envolvido em vendas e o output do modelo anterior, passageiro.km oferecidos. O output dá-se em passageiro.km pagos. A baixa eficiência neste modelo demonstra a ineficiência da sistemática de vendas da empresa em relação aos vôos por ela realizados e aos meios disponíveis para a venda. Implicitamente, este modelo considera uma abordagem semelhante ao Fator de Ocupação, indicador largamente utilizado no mercado, mas combatido pelas abordagens mercadológicas inerentes ao transporte aéreo, como a segmentação da demanda.

O terceiro modelo (M3) obtém-se da justaposição dos dois anteriores e busca retratar a eficiência das operações de negócios da empresa, ou seja, eficiência conjunta de uso da frota

Pesquisa Operacional, v.23, n.2, p.325-345, Maio a Agosto de 2003 
e venda da capacidade oferecida. Por isso, é chamado de modelo global. Nele consideram-se como inputs combustível usado, soma das capacidades de passageiros do total das aeronaves da frota e quantidade total de pessoal, e o output dá-se em passageiro.km pagos, o mesmo do modelo de mercado.

A Figura 1 mostra esquematicamente os três modelos e suas relações.

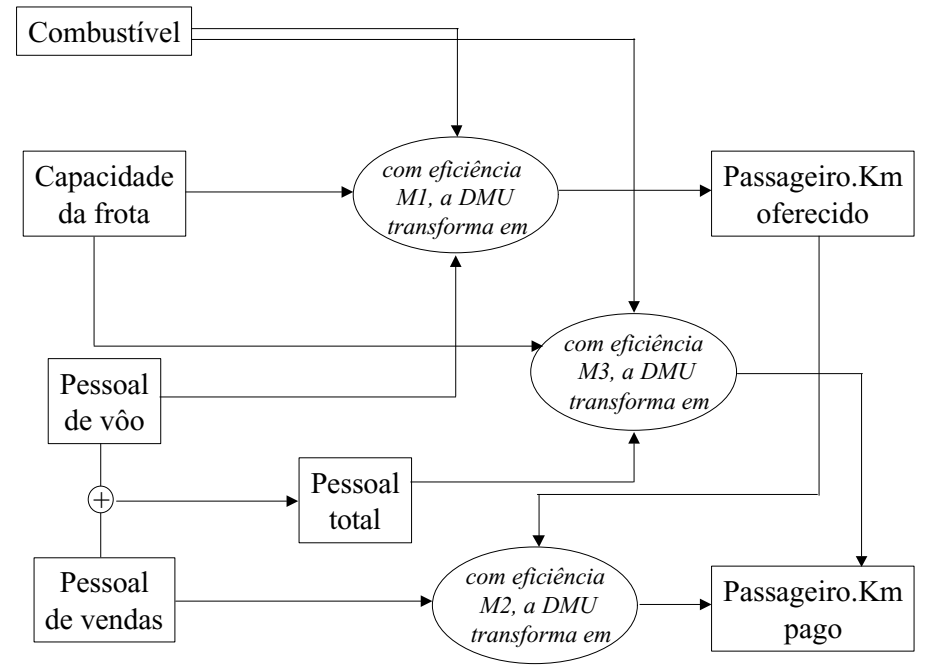

Figura 1 - Modelos DEA usados e seus relacionamentos.

\subsection{Resultados}

A Tabela 1 resume as eficiências obtidas pelos três modelos; os dados utilizados como inputs e outputs encontram-se disponíveis com os autores. A análise e a interpretação dos resultados para os modelos operacional, de vendas e global, bem como o estudo do modelo global para os grandes grupos nacionais encontram-se nos itens que se seguem.

Tabela 1 - Eficiências das DMUs analisadas nos três modelos.

\begin{tabular}{cccc}
\hline \multirow{2}{*}{ DMUs } & \multicolumn{3}{c}{ Eficiência (\%) } \\
\cline { 2 - 4 } & Operacional & de Vendas & Global \\
\hline Abaeté 1998 & 100,00 & 71,80 & 100,00 \\
Abaeté 1999 & 83,83 & 60,34 & 83,45 \\
Abaeté 2000 & 83,73 & 48,02 & 82,44 \\
Grupo Tam 1998 & 68,01 & 92,62 & 99,34 \\
Grupo Tam 2000 & 39,26 & 74,81 & 90,82 \\
Grupo Tam 99 & 76,91 & 87,59 & 93,36 \\
Grupo Tba 1998 & 97,72 & 78,78 & 87,32 \\
Grupo Tba 1999 & 73,15 & 62,83 & 70,16 \\
Grupo Tba 2000 & 83,39 & 68,99 & 83,09 \\
Grupo Varig 1998 & 100,00 & 100,00 & 100,00 \\
Grupo Varig 1999 & 100,00 & 83,41 & 87,05 \\
Grupo Varig 2000 & 100,00 & 91,39 & 98,69 \\
Helisul 1998 & 37,82 & 72,81 & 32,48 \\
Helisul 1999 & 21,25 & 80,31 & 37,86
\end{tabular}




\begin{tabular}{|c|c|c|c|}
\hline Interbrasil 1998 & 49,67 & 70,21 & 34,70 \\
\hline Interbrasil 1999 & 52,97 & 66,42 & 46,64 \\
\hline Interbrasil 2000 & 41,50 & 80,79 & 39,87 \\
\hline Itapemirim 1998 & 100,00 & 100,00 & 100,00 \\
\hline Meta 1999 & 91,33 & 53,37 & 89,18 \\
\hline Meta 2000 & 61,80 & 56,35 & 57,57 \\
\hline \multirow{2}{*}{$D M U s$} & \multicolumn{3}{|c|}{ Eficiência (\%) } \\
\hline & Operacional & de Vendas & Global \\
\hline Nordeste 1998 & 72,64 & 69,88 & 56,55 \\
\hline Nordeste 1999 & 55,02 & 69,19 & 60,34 \\
\hline Nordeste 2000 & 47,85 & 71,49 & 57,59 \\
\hline Pantanal 1998 & 60,67 & 53,47 & 40,32 \\
\hline Pantanal 1999 & 25,07 & 31,14 & 27,32 \\
\hline Pantanal 2000 & 28,32 & 40,26 & 28,19 \\
\hline Passaredo 1998 & 99,98 & 100,00 & 100,00 \\
\hline Passaredo 1999 & 73,70 & 97,20 & 100,00 \\
\hline Passaredo 2000 & 54,22 & 51,09 & 49,27 \\
\hline Penta 1998 & 47,04 & 57,72 & 34,40 \\
\hline Penta 1999 & 29,15 & 100,00 & 35,92 \\
\hline Penta 2000 & 34,57 & 95,71 & 36,50 \\
\hline Presidente 1998 & 70,91 & 59,68 & 72,16 \\
\hline Presidente 1999 & 54,57 & 88,82 & 53,33 \\
\hline Rico 1998 & 89,26 & 65,02 & 66,24 \\
\hline Rico 1999 & 96,92 & 70,16 & 100,00 \\
\hline Rico 2000 & 44,68 & 69,84 & 68,58 \\
\hline Rio-Sul 1998 & 63,05 & 78,75 & 65,42 \\
\hline Rio-Sul 1999 & 41,07 & 80,56 & 67,80 \\
\hline Rio-Sul 2000 & 36,51 & 82,36 & 69,14 \\
\hline Taba 1998 & 39,72 & 58,05 & 35,01 \\
\hline Taba 1999 & 11,05 & 57,92 & 28,51 \\
\hline Taf 2000 & 21,78 & 100,00 & 33,18 \\
\hline Taf 1998 & 51,13 & 43,69 & 48,12 \\
\hline Taf 1999 & 19,93 & 61,75 & 24,26 \\
\hline Tam Linhas 2000 & 22,10 & 70,52 & 58,61 \\
\hline Tam Meridionais 1998 & 100,00 & 98,42 & 100,00 \\
\hline Tam Meridionais 1999 & 100,00 & 69,10 & 100,00 \\
\hline Tam Meridionais 2000 & 51,67 & 76,18 & 96,48 \\
\hline Tam Regionais 1998 & 59,90 & 88,70 & 86,96 \\
\hline Tam Regionais 1999 & 42,46 & 87,48 & 87,16 \\
\hline Tam Regionais 2000 & 37,87 & 76,99 & 70,93 \\
\hline Tavaj 1998 & 30,70 & 74,72 & 21,70 \\
\hline Tavaj 1999 & 19,24 & 71,53 & 21,90 \\
\hline Tavaj 2000 & 30,18 & 63,20 & 29,90 \\
\hline Total 1998 & 48,00 & 80,48 & 51,81 \\
\hline Total 1999 & 25,38 & 87,69 & 46,24 \\
\hline Total 2000 & 21,53 & 100,00 & 31,81 \\
\hline Transbrasil 1998 & 100,00 & 78,81 & 87,86 \\
\hline Transbrasil 1999 & 77,93 & 62,69 & 70,93 \\
\hline Transbrasil 2000 & 92,81 & 68,83 & 84,06 \\
\hline Trip 1999 & 39,18 & 98,54 & 69,09 \\
\hline Trip 2000 & 44,42 & 100,00 & 64,10 \\
\hline Varig 1998 & 100,00 & 100,00 & 100,00 \\
\hline Varig 1999 & 92,95 & 84,08 & 87,75 \\
\hline Varig 2000 & 94,46 & 93,04 & 100,00 \\
\hline
\end{tabular}




\begin{tabular}{cccc} 
Vasp 1998 & 100,00 & 100,00 & 100,00 \\
Vasp 1999 & 85,07 & 65,20 & 63,29 \\
Vasp 2000 & 69,40 & 75,49 & 68,88 \\
\hline
\end{tabular}

\subsubsection{Modelo Operacional}

Este modelo objetiva verificar a capacidade de produção (assento.km) de cada companhia aérea. Em uma primeira análise dos resultados do modelo foram classificadas 10 companhias como eficientes. Destas 10, 8 só o foram pelo fato de o PPL do modelo DEA VRS ter atribuído, na solução ótima, valor extremamente elevado ao peso do input combustível, desconsiderando quase por completo as demais variáveis.

A maioria das empresas manteve as características de frota praticamente constantes no período, estando o consumo específico de combustível em um patamar similar nos anos em análise. Essa característica de influência, diretamente proporcional do consumo de combustível e produção, aliada à característica dos modelos DEA clássicos em poder atribuir pesos zero a algumas variáveis, fez com que se pensasse em retirar a variável combustível do modelo.

O uso da variável combustível demonstra-se importante em qualquer modelo de transporte, já que é a fonte de energia que movimenta as frotas. Assim, a retirada da variável, considerada atitude extrema por subtrair informação do modelo, foi substituída pela imposição de um limite ao peso máximo que a variável pode ter, também pelo fato de os padrões operacionais definidos pelos fabricantes das aeronaves serem muito similares.

A limitação à importância de uma variável pode ser feita de várias formas diferentes. Novaes (2001b) usa o método de cone ratio em um estudo sobre metrô. Neste artigo, devido ao software usado foi preferido o método de impor restrição à participação da variável na formação do input virtual. Os resultados apresentados anteriormente (Tabela 1) incorporam uma restrição de $42,55 \%$ ao input combustível. Com esse valor, não foi alterado o número de DMUs eficientes no modelo, embora tenha sido alterada a ordenação das DMUs eficientes. Qualquer valor acima do usado diminuiria o número de DMUs eficientes.

As companhias eficientes nesse modelo são: Tam Meridionais-1998, Transbrasil-1998, Varig-1998, Vasp-1998, Abaeté-1998, Itapemirim-1998, Grupo Varig-1998, Tam Meridionais-1999, Grupo Varig-1999, Grupo Varig-2000. Dentre estas, Abaeté-1998 e Itapemirim-1998 demonstram-se eficientes somente por estarem isoladas na escala em que operam (possuem o menor valor de um dos inputs, pessoal de vôo e capacidade de passageiros, respectivamente).

Pelo fato de estar em consideração não um modelo de mercado mas um modelo que retrata as operações das companhias, não se espera que este modelo detecte diretamente mudanças na economia. No entanto, o número de DMUs eficientes em cada ano sugere uma certa inércia em reagir à crise de 1999, verificada pela redução do número de DMUs eficientes de 1998 para 1999. Ao contrário do que seria esperado, em 2000 não há aumento do número de DMUs operacionalmente eficientes. Uma possível explicação consiste no fato de as empresas terem vislumbrado o final da crise e, portanto, terem começado a se re-equipar antes de efetivamente aumentarem sua produção.

Três casos particulares merecem destaque. A TAF no modelo operacional teve eficiências para os anos de 1998, 1999 e 2000, respectivamente, de 40,25\%, 19,54\%, 21,50\%, em contraste com a alta eficiência do modelo de vendas. Isso sugere que essa alta eficiência foi 
obtida não por "vender muito", mas por "voar pouco". O Grupo Tam mostra-se operacionalmente pouco eficiente e essa eficiência é decrescente (47,29\% em 1998 a 22,09\% em 2000). Uma possível razão consiste na já mencionada mudança de características do grupo no período em análise. Em contrapartida, nota-se um padrão constante de eficiência do Grupo Varig ao longo do tempo.

\subsubsection{Modelo de Vendas}

Este modelo busca analisar a eficiência das companhias aéreas no que se refere à capacidade de comercializar sua produção, não considerando as características operacionais. Inicialmente foram apontadas 9 DMUs como sendo eficientes. No entanto, 3 destas (Penta1999, Total-2000, Trip-2000) tiveram seu resultado distorcido por terceirizar a venda, o que acarreta valor nulo para a variável quantidade de pessoal de vendas. Restaram, assim, 6 DMUs realmente eficientes (Grupo Varig-1998, Itapemirim-1998, Passaredo-1998, Varig1998, Vasp-1998, Taf-2000).

Observa-se que destas 6 DMUs, 5 são eficientes no ano de 1998, nenhuma em 1999 e apenas $1 \mathrm{em}$ 2000. Tal fato sugere uma grande dificuldade de vendas em 1999 com início de recuperação em 2000, refletindo a crise de 1999 provocada pela desvalorização cambial.

A companhia TAF, única eficiente em 2000, vinha obtendo eficiências bastante baixas nos anos anteriores, com grande redução de atividades em 1999, levando alguns analistas a prognosticar o fim de suas atividades. No entanto, a empresa apresentou sinais de recuperação, inclusive com a diversificação de sua frota com a adição de uma aeronave de grande porte (Lucchesi, 2001), usada em transporte de carga para a Empresa Brasileira de Correios e Telégrafos. Observa-se, ainda, que de 1999 para 2000, embora continuasse a redução de oferta de lugares, houve o aumento das vendas, com o conseqüente aumento de eficiência. Tal fato pode em parte ser atribuído ao fim da suplementação tarifária (DAC, 1992; DAC, 1998), obrigando a empresa a buscar adequação à realidade do mercado. A Figura 2 representa graficamente os resultados da companhia TAF, nos três anos em estudo, mostrando apenas as variáveis mais significativas.
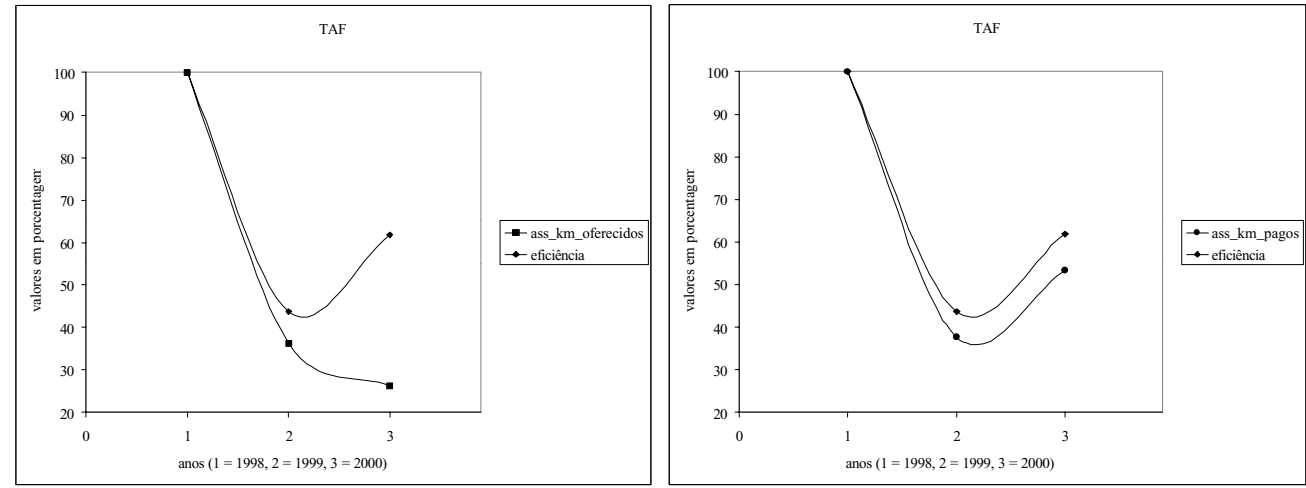

Figura 2 - Resultados da companhia aérea TAF no modelo de vendas. 
Os resultados do modelo de vendas ainda permitem a análise do comportamento dos grandes grupos nacionais durante o período de crise. Assim, no médio prazo, os grupos Varig e Transbrasil têm uma adequação positiva frente à crise. No caso do Grupo Transbrasil para que não sejam tiradas conclusões pouco fundamentadas, seria importante analisar alguns dados não constantes deste modelo, como por exemplo, as tarifas praticadas e a retração de atividades deste grupo. Os dados do Grupo Varig mostram uma tendência a acompanhar os acontecimentos econômicos, ou seja, tanto a oferta quanto à "eficiência de vendas" apresentam queda durante a crise de 1999, mostrando recuperação no período seguinte.

No curto prazo, o Grupo Tam suporta melhor a crise, aparentando entrar em situação pior em seguida. Entretanto, essa aparente piora explica-se pela mudança de foco do grupo, que deixou de ser regional para tornar-se nacional e internacional, com entrada em operação de aviões maiores em rotas que estavam sendo prospectadas.

A companhia Vasp apresenta o pior comportamento dentre os grandes grupos, tanto a curto quanto a médio prazo. A Tabela 2 mostra os resultados das quedas de eficiência dos grandes grupos nacionais para o modelo que retrata as vendas.

Tabela 2 - Quedas de eficiência dos grandes grupos nacionais no modelo de vendas.

\begin{tabular}{ccc}
\hline \multirow{2}{*}{ Companhias } & \multicolumn{2}{c}{ Queda de eficiência (\%) } \\
\cline { 2 - 3 } & 1998 para 1999 & 1998 para 2000 \\
\hline Grupo Varig & 17 & 9 \\
Grupo Tam & 5 & 18 \\
Grupo Transbrasil & 16 & 10 \\
Vasp & 35 & 25 \\
\hline
\end{tabular}

Além dos grandes grupos nacionais, mostra-se interessante analisar a companhia Passaredo, pois apresenta uma grande queda de eficiência posterior ao período de crise (Figura 3), que pode ser explicado pelo fim das operações do Airbus A310 que operava em regime semelhante ao de charter. A empresa continuou a operar com aviões EMB120-Brasília nas rotas de baixo volume de tráfego.

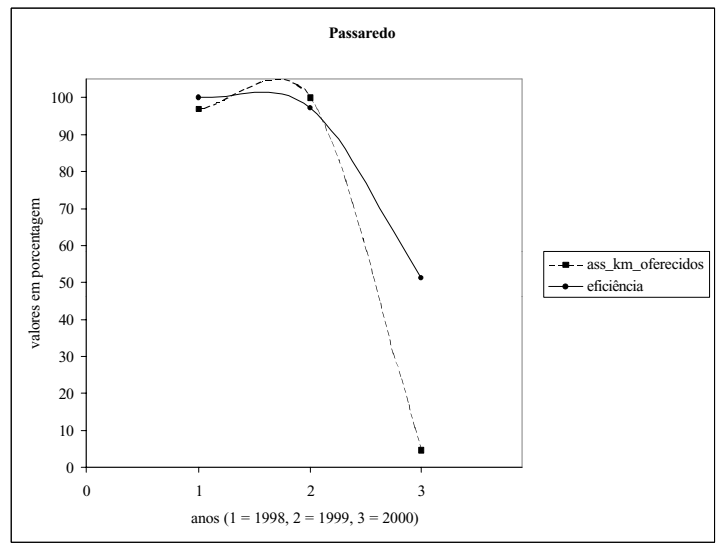

Figura 3 - Resultados da companhia aérea Passaredo. 


\subsubsection{Modelo Global}

O modelo global pretende agregar em uma só análise as informações dos modelos anteriores, e faz uma comparação entre os meios de produção e os resultados das vendas. Ao contrário do modelo operacional, não foi necessária a adição de restrição ao peso da variável combustível, já que das 11 DMUs eficientes apenas 4 apresentaram valor alto de peso para essa variável.

As 11 DMUs globalmente eficientes são: Tam Meridionais-1998, Varig-1998, Vasp-1998, Abaeté-1998, Passaredo-1998, Itapemirim-1998, Grupo Varig-1998, Tam Meridionais-1999, Passaredo-1999, Rico-1999, Varig-2000.

Devido a terem menor valor no input quantidade de pessoal total - Abaeté-1998 - e nos inputs capacidade de passageiros e combustível utilizado - Itapemirim-1998 -, estas DMUs são consideradas eficientes independentemente do nível de output atingido. Para verificar se devem ser consideradas realmente eficientes foram introduzidas restrições crescentes aos pesos dos inputs mencionados. Foi observado que à medida que se diminuía o máximo valor admissível do peso dos inputs capacidade de passageiros e combustível, a DMU Itapemirim1998 permanecia eficiente. A partir de um valor de peso próximo a 50\%, Rico-1999, Varig2000 e Tam Meridionais-1999 deixavam de ser eficientes. Para o caso da DMU Abaeté foi adotado procedimento semelhante, diferindo-se do resultado, já que quando se aumentou a restrição ao peso do input quantidade de pessoal total, essa DMU foi a primeira a sair do grupo das eficientes. Assim, conclui-se que a DMU Itapemirim-1998 é realmente eficiente, enquanto que Abaeté-1998 pode ser considerada falsamente eficiente. Cabe ressaltar que apesar de a DMU Itapemirim-1998 aparecer como eficiente, essa eficiência é meramente técnica (Cooper et al., 2000), embora operasse em uma escala ineficiente.

A companhia Passaredo foi eficiente em 1998 e 1999, mesmo usando o modelo DEA que não considera os retornos de escala, apresentando-se em situação diversa da companhia Itapemirim em 1998, já que opera com eficiência técnica (vôos de rotas de médio curso são geralmente mais eficientes que vôos regionais, em especial em relação ao item combustível). Ao devolver o Airbus A310, a companhia Passaredo não só perdeu sua eficiência técnica como passou a operar em uma escala ineficiente. Em 2000, sua eficiência global foi de 49,27\%.

Um resultado inesperado consiste no da eficiência da DMU Rico-1999, por ser uma companhia de pouca repercussão e, principalmente, pelo fato de 1999 ser o ano auge da crise cambial. Essa companhia, que opera na região amazônica, beneficia-se das características da região onde o transporte aéreo tem importância vital (Lucchesi, 2001). Além disso, graças à utilização do Cessna Caravan anfíbio, a empresa entrou no pouco explorado mercado do Eco-turismo e do transporte de pescadores, compensando largamente os efeitos da crise. Analisando-se os resultados, verifica-se que a eficiência dessa DMU deve-se essencialmente à relação entre passageiro.km pagos e capacidade de passageiros, ignorando a quantidade de pessoal total.

\subsubsection{Análise dos grandes grupos nacionais no modelo global}

Analisando-se a eficiência das várias DMUs constituintes dos grupos, pode-se identificar as DMUs de maior impacto para a eficiência do grupo. Para o Grupo Varig nos três anos considerados, a própria Varig foi sempre pelo menos tão eficiente quanto o grupo. Rio-Sul e

Pesquisa Operacional, v.23, n.2, p.325-345, Maio a Agosto de 2003 
Nordeste, nesta ordem, apresentam eficiências menores. Mesmo com o uso do modelo DEA CRS, que não considera efeitos de escala, a Varig demonstra-se mais eficiente que as coligadas.

Para o Grupo Tam, devido à reestruturação interna ocorrida em 2000, não é possível uma análise geral naquele período. Nos anos de 1998 e 1999, a empresa Tam Regionais apresentava a maior eficiência dentro do grupo.

O Grupo Transbrasil e a própria Transbrasil apresentam, em todos os anos, eficiências muito semelhantes. Os valores de eficiência da Interbrasil praticamente não afetam o grupo, devido à escala de operação muito menor. A Figura 4 mostra a eficiência e um input do modelo de vendas para este grupo. Nota-se que o grupo foi seriamente atingido pela crise de 1999, com recuperação em 2000. Entretanto, esta análise não considera os preços extremamente baixos que o grupo praticou nesse período, sendo ainda possível observar uma grande redução na escala de operação da companhia. Para uma companhia altamente endividada isto significa que dívidas contraídas em grande escala deveriam ser pagas por uma companhia operando em pequena escala. Esses dois fatos juntos podem ser uma das explicações para a suspensão das atividades do grupo em dezembro de 2001.

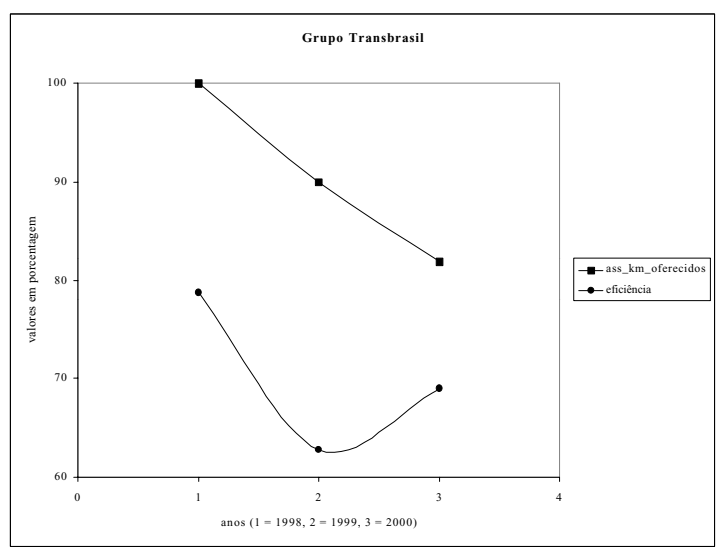

Figura 4 - Resultados para o Grupo Transbrasil.

A Tabela 3 mostra os resultados dos grandes grupos nacionais no modelo global, que são coerentes com os apresentados na Tabela 1 . O modelo global ressalta a recuperação quase completa do Grupo Varig no ano 2000, além de mostrar uma pequena recuperação do Grupo Tam, não perceptível no modelo de vendas.

Tabela 3 - Quedas de eficiência dos grandes grupos nacionais no modelo global.

\begin{tabular}{ccc}
\hline \multirow{2}{*}{ Companhias } & \multicolumn{2}{c}{ Queda de eficiência (\%) } \\
\cline { 2 - 3 } & 1998 para 1999 & 1998 para 2000 \\
\hline Grupo Varig & 13 & 2 \\
Grupo Tam & 9 & 6 \\
Grupo Transbrasil & 17 & 6 \\
Vasp & 37 & 31 \\
\hline
\end{tabular}




\section{Análise de Alvos}

A determinação de benchmarks é uma das características gerenciais mais importantes dos modelos DEA clássicos. Na área de transportes coletivos, por exemplo, Novaes (2001b) analisa o benchmarking de metrôs não eficientes, com ênfase ao metrô de São Paulo. Ainda na área de transportes, Novaes (2001a) apresenta um modelo de benchmarks para operadores logísticos. Esses trabalhos usam como benchmark o alvo radial calculado nos modelos DEA clássicos.

Entretanto, o alvo de cada DMU ineficiente calculado pelos modelos DEA clássicos pode ser considerado não adequado às políticas gerenciais das companhias. $\mathrm{O}$ alvo único é, portanto, uma limitação à atividade gerencial. Torna-se, assim, interessante contar com alvos alternativos que confiram um maior grau de liberdade ao gestor. Existem na literatura vários exemplos de uso de alvos alternativos, como em Portela et al. (2002), Soares de Mello et al. (2002), Soares de Mello (2002). Neste artigo é usado um modelo de Programação Linear Multiobjetivo, cuja fronteira eficiente é idêntica à do modelo DEA (Angulo Meza \& Lins, 2002; Angulo Meza, 2002), que é uma evolução dos modelos de Thanassoulis \& Dyson (1992) e Zhu (1996). O número de alvos encontrados para cada DMU ineficiente varia com a posição da DMU em relação à fronteira.

O modelo de Programação Linear Multiobjetivo utilizado visa independência na maximização dos outputs e na minimização dos inputs, de tal forma que seja encontrado um conjunto de metas na fronteira eficiente. Devido ao fato de incorporar as opiniões dos decisores na escolha do alvo dentro de um conjunto, o modelo não deve ser usado conjuntamente com outras técnicas de explicitação de preferências.

A aplicação do modelo multiobjetivo é feita apenas para o modelo de vendas, por ser o mais simples e o que melhor identifica a crise cambial de 1999. A formalização desse modelo, para o estudo de caso deste artigo, é dada por (2), onde $\phi_{1}$ é o fator de incremento da variável output, $\varphi_{1}, \varphi_{2}$ são os fatores de decremento das variáveis input, $x_{1 j}, x_{2 j}$ e $y_{i j}$, são os valores das variáveis de input e o output para a DMU $j, \lambda_{j}$ são os coeficientes da combinação linear convexa que representam os benchmarks, isto é, as companhias aéreas eficientes, para a $\operatorname{DMU} j$.

As funções objetivo consideram variações independentes para cada variável, neste caso maximizam o output passageiro.km pago (2.1) e minimizam os inputs pessoal de vendas (2.2) e passageiro.km oferecido (2.3), e estão sujeitas a que o aumento na variável output (2.4) e o decremento nas variáveis input (2.5) e (2.6) sejam até a fronteira eficiente, determinada pelas companhias eficientes. A restrição (2.7) garante a existência de retornos variáveis de escala. Por último, as restrições (2.8), (2.9) e (2.10) determinam que, para o alvo calculado, a produção não diminua nem os recursos aumentem, respectivamente, em relação aos valores originais da companhia.

Devido às restrições (2.8), (2.9) e (2.10) esse modelo é chamado de modelo com dominância - MORO-D-VRS (Angulo Meza, 2002). Como uma interpretação menos formal desse modelo, pode-se afirmar que a DMU evita perder suas características de operação na busca do alvo. Se forem retiradas essas restrições, o modelo é dito sem dominância - MORO-VRS (Angulo Meza, 2002), o que significa que a DMU na busca de seu alvo pode aumentar seus inputs ou diminuir seus outputs, já que pode ser projetada em qualquer ponto da fronteira Pareto eficiente. 
O uso de uma formulação multiobjetivo garante que os alvos nunca serão encontrados na região da fronteira que apresenta ineficiência de Pareto, tornando assim desnecessário a incorporação de folgas à função objetivo. Por outro lado, as restrições (2.4), (2.5) e (2.6), que obrigam o alvo a estar situado na fronteira, são restrições de igualdade para as quais as folgas são nulas. Assim, nesse modelo não faz sentido o uso de folgas, tão necessárias em outros modelos DEA, já que estas consideram a hipótese da projeção equiproporcional das variáveis.

$$
\begin{aligned}
& \max \phi_{1} \\
& \min \varphi_{1} \\
& \min \varphi_{2} \\
& \text { sujeito a } \\
& \phi_{1} y_{1 j_{0}}=\sum_{j=1}^{n} y_{1 j} \lambda_{j} \\
& \varphi_{1 i} x_{1 j_{0}}=\sum_{j=1}^{n} x_{1 j} \lambda_{j} \\
& \varphi_{2 i} x_{2 j_{0}}=\sum_{j=1}^{n} x_{2 j} \lambda_{j} \\
& \sum_{j=1}^{n} \lambda_{j}=1 \\
& \phi_{1} \geq 1 \\
& \varphi_{1} \leq 1 \\
& \varphi_{2} \leq 1
\end{aligned}
$$

Uma DMU é eficiente se, e somente se, é encontrada uma única solução não dominada com $\phi_{1}^{*}=\varphi_{1}^{*}=\varphi_{2}^{*}=1$; caso contrário, uma ou mais soluções não dominadas podem ser encontradas. Um alvo para uma $\mathrm{DMU}_{0}$ ineficiente é dado por (3) e é válido para os modelos MORO com ou sem dominância.

$$
\begin{aligned}
& y_{1 j_{0}}^{*}=\phi_{r}^{*} y_{1 j_{0}} \\
& x_{1 j_{0}}^{*}=\varphi_{1}^{*} x_{1 j_{0}} \\
& x_{2 j_{0}}^{*}=\varphi_{2}^{*} x_{2 j_{0}}
\end{aligned}
$$

No estudo de caso deste artigo foi escolhido o modelo com dominância (MORO-D-VRS) para impedir que uma companhia aérea regional, por exemplo TAF, buscasse como alvo uma grande companhia aérea internacional, por exemplo Varig, ou vice-versa.

As Figuras 5 e 6 ilustram um exemplo didático com as possíveis projeções de uma DMU ineficiente segundo os modelos MORO-D-VRS e MORO-VRS. Na Figura 5, modelo MORO-D-VRS, a DMU $o$ pode ser projetada em qualquer ponto entre $a$ e $b$. Na Figura 6, modelo MORO-VRS, a projeção da DMU o estará situada entre A e C, ou seja, em toda a fronteira Pareto eficiente. 
Na solução de modelos multiobjetivo têm-se dois tipos de métodos de solução. Um encontra soluções básicas não dominadas; outro permite percorrer a fronteira não dominada. $\mathrm{O}$ primeiro desses métodos eqüivale em DEA a considerar como possíveis alvos as DMUs extremo-eficientes e DMU virtuais que limitam a região viável de projeção (pontos $\mathrm{B}, a$ e $b$, respectivamente, da Figura 5). O segundo eqüivale a percorrer de forma interativa a região de projeção, obrigatoriamente Pareto eficiente.

Quando a fronteira é percorrida de forma interativa, encontra-se uma infinidade de possíveis alvos e, portanto, precisa-se da intervenção do especialista para a escolha de um deles. Já no primeiro caso, conta-se com um conjunto finito de possíveis alvos, o que possibilita uma análise global dessas alternativas. Essa foi a abordagem utilizada neste artigo.

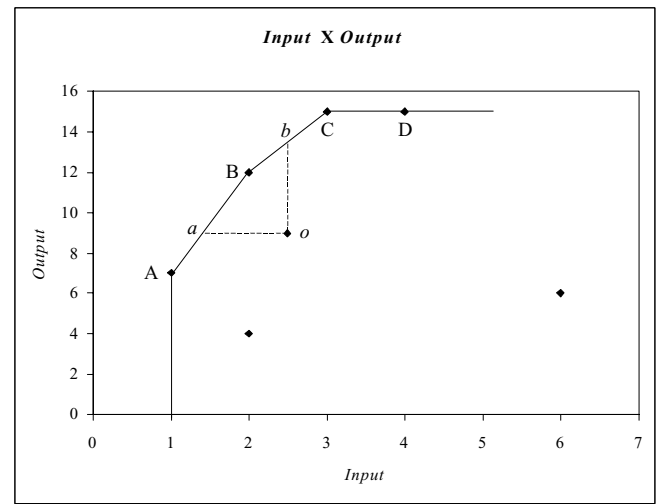

Figura 5 - Exemplo de projeções no modelo MORO-D-VRS.

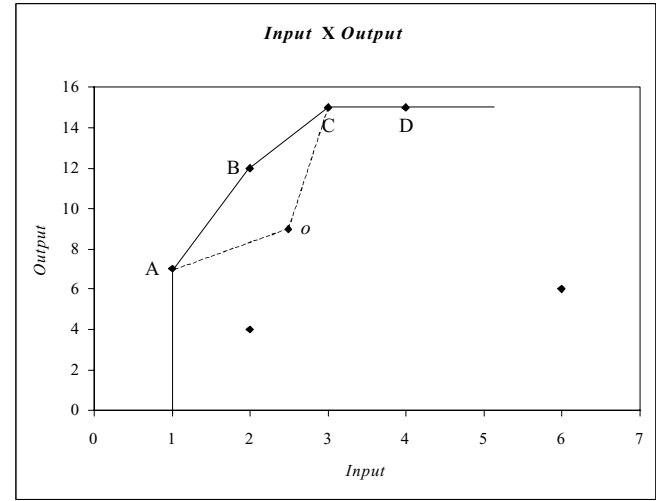

Figura 6 - Exemplo de projeções no modelo MORO-VRS.

\subsection{Resultados do modelo MORO-D-VRS}

A aplicação do modelo (2) revelou um conjunto de metas para cada companhia aérea, permitindo uma flexibilidade gerencial (Tabela 4), e que a vasta maioria das companhias aéreas nos anos de 1999 e 2000 têm como metas companhias no ano de 1998. Algumas 
companhias nos anos de 1999 e 2000 tinham como meta elas mesmas no ano de 1998. Essa é uma situação que fornece diretrizes impossíveis de serem aplicadas na prática, já que a situação do mercado de 1999 era muito pior do que a do ano de 1998, como conseqüência da mudança na política cambial ocorrida em janeiro de 1999. Ainda assim, alguns resultados interessantes podem ser destacados.

As DMUs TAF no ano de 1998 (Taf 1998) e TAF no ano de 1999 (Taf 1999), têm como principal referência a DMU Taf 2000, o que indica que, ao considerar-se estritamente o aspecto de vendas, a empresa foi gerida na direção de seu alvo.

O Grupo Varig em 1999 e 2000 apresenta como principal referência a companhia VARIG 1998. Apesar disto ressaltar uma situação anterior à crise cambial, é interessante verificar que o Grupo Varig apresenta como referência a companhia Varig isoladamente sem as subsidiárias Rio Sul e Nordeste.

O Grupo Transbrasil em 1998 e em 1999 tem como metas principais as DMUs Vasp 1998, Passaredo 1998 e Total 2000 e, em menor participação, PENTA 1999. Observa-se que, excetuando-se a DMU Vasp 1998, as outras duas apresentaram sérios problemas. A companhia Passaredo, ao deixar do operar os aviões do tipo Airbus, tornou-se uma companhia de porte muito pequeno, inexpressiva no mercado. A companhia Total deixou de operar vôos regulares de passageiros. Já a companhia Penta é de porte extremamente pequeno e a sua presença no conjunto de alvos indica a redução de tamanho que a Transbrasil sofreu. Embora DEA seja um modelo de análise e não de previsão, um estudo precoce dessa situação poderia antecipar o diagnóstico da crise enfrentada pela Transbrasil.

Tabela 4 - Número de metas obtidas para cada companhia aérea ineficiente.

\begin{tabular}{cc}
\hline DMU & Número de alvos obtidos \\
\hline Grupo TAM 1998 & 3 \\
Grupo TAM 1999 & 8 \\
Grupo TAM 2000 & 8 \\
Grupo Transbrasil 1998 & 9 \\
Grupo Transbrasil 2000 & 9 \\
Grupo Transbrasil 1999 & 9 \\
Grupo Varig 1999 & 4 \\
Grupo Varig 2000 & 4 \\
Passaredo 1999 & 5 \\
Passaredo 2000 & 6 \\
TAF 1998 & 6 \\
TAF 1999 & 7 \\
Vasp 1999 & 8 \\
Vasp 2000 & 8 \\
\hline
\end{tabular}

Ao considerar-se o fato já citado de muitas companhias terem alvos no ano de 1998, as metas foram reavaliadas usando-se somente as DMUs correspondentes aos anos de 1999 e 2000. Convém ressaltar que, devido ao fato de DEA calcular eficiências relativas, a retirada dos dados de 1998 fez com que algumas DMUs de 1999 e 2000 passassem a ser eficientes. Os resultados desse modelo fornecem informações mais úteis por tratarem de anos com panorama macroeconômico homogêneo. 
A Tabela 5 mostra os alvos alternativos para algumas companhias aéreas ineficientes dentre as analisadas. Os percentuais referem-se à contribuição de cada DMU eficiente na formação do alvo da DMU ineficiente.

A DMU Taf 1999 repete como referência principal a DMU Taf 2000, ratificando a correta evolução dessa companhia no que se refere à política de vendas.

O Grupo Transbrasil, tanto em 1999 quanto em 2000, apresenta uma grande variedade de alvos o que indica uma falta de definição de rumo a ser seguido. Dentre estes alvos, a referência principal é a DMU Vasp 2000. Em relação às demais referências, a principal é a DMU Vasp 1999, ou seja a Transbrasil deveria ter-se espelhado nas práticas gerenciais da Vasp. Cabe destacar que a VASP também já havia passado por uma crise de liquidez e por uma drástica redução de frota, com caraterísticas iniciais semelhantes às que viriam a ocorrer com a Transbrasil. O fato de a companhia Vasp ainda se apresentar como uma das grandes companhias aéreas nacionais, juntamente com os resultados aqui indicados, sugere a necessidade de um estudo comparativo das estratégias adotadas pela Vasp e Transbrasil para enfrentar as respectivas crises.

O Grupo VARIG 1999 apresenta como principais referências o Grupo Varig 2000 e a Varig 2000. Tal fato, além de sugerir que houve uma correta gestão no período de crise cambial, reforça a indicação anterior de que as coligadas Rio Sul e Nordeste são um peso para o grupo, ao considerar-se apenas a eficiência de vendas.

A DMU Passaredo 2000 apresenta como referências importantes as DMUs Presidente 1999, Total 2000, Trip 1999 e Passaredo 1999. A companhia Presidente deixou de operar; a empresa Total deixou de operar regularmente; a companhia Passaredo em 1999 operava em escala maior que a atual (Flap, 2001). Resta como única referência aceitável, a DMU Trip 1999. Esses resultados sugerem que, no tocante à política de vendas, a diminuição de escala da companhia Passaredo não foi uma boa estratégia e não sugerem um futuro promissor.

Tabela 5 - Alvos alternativos para as companhias aéreas ineficientes.

\begin{tabular}{|c|c|c|c|c|c|c|}
\hline & $\begin{array}{c}\text { Taf } \\
1999\end{array}$ & $\begin{array}{c}\text { Grupo } \\
\text { Transbrasil } \\
1999\end{array}$ & $\begin{array}{c}\text { Grupo } \\
\text { Transbrasil } \\
2000\end{array}$ & $\begin{array}{c}\text { Grupo Varig } \\
1999\end{array}$ & $\begin{array}{c}\text { Passaredo } \\
2000\end{array}$ & $\begin{array}{l}\text { Grupo Tam } \\
2000\end{array}$ \\
\hline $\begin{array}{c}\text { Alvo } \\
I\end{array}$ & $\begin{array}{l}\text { Presidente } \\
\text { 1999: 36,1\% } \\
\text { Taf 2000: } \\
63,9 \%\end{array}$ & $\begin{array}{l}\text { Passaredo } \\
\text { 1999: } 23,0 \% \\
\text { Varig 2000: } \\
8,6 \% \\
\text { Vasp 2000: } \\
68,4 \% \\
\end{array}$ & $\begin{array}{l}\text { Passaredo } \\
\text { 1999: 28,0\% } \\
\text { Varig 2000: } \\
7,0 \% \\
\text { Vasp 2000: } \\
65,0 \%\end{array}$ & $\begin{array}{l}\text { Grupo Varig } \\
2000: 74,8 \% \\
\text { Varig 2000: } \\
23,2 \% \\
\text { Vasp 1999: } \\
2,0 \%\end{array}$ & $\begin{array}{l}\text { Passaredo } \\
\text { 1999: 4,4\% } \\
\text { Presidente } \\
\text { 1999: } 81,4 \% \\
\text { Taf 2000: } \\
14,2 \%\end{array}$ & $\begin{array}{l}\text { Varig 2000: } \\
16,9 \% \\
\text { Vasp 2000: } \\
51,0 \% \\
\text { Vasp 1999: } \\
32,1 \% \\
\end{array}$ \\
\hline $\begin{array}{c}\text { Alvo } \\
\text { II }\end{array}$ & $\begin{array}{l}\text { Passaredo } \\
\text { 1999: 1,00\% } \\
\text { Taf 2000: } \\
99,9 \%\end{array}$ & $\begin{array}{l}\text { Varig 2000: } \\
2,2 \% \\
\text { Vasp 2000: } \\
97,8 \%\end{array}$ & $\begin{array}{l}\text { Passaredo } \\
\text { 1999: 2,7\% } \\
\text { Vasp 2000: } \\
97,3 \%\end{array}$ & $\begin{array}{l}\text { Grupo Varig } \\
\text { 2000: } 87,0 \% \\
\text { Vasp 1999: } \\
13,0 \%\end{array}$ & $\begin{array}{l}\text { Passaredo } \\
\text { 1999: 4,4\% } \\
\text { Presidente } \\
\text { 1999: } 95,6 \%\end{array}$ & $\begin{array}{l}\text { Varig 2000: } \\
15,9 \% \\
\text { Vasp 2000: } \\
47,5 \% \\
\text { Vasp 1999: } \\
36,6 \% \\
\end{array}$ \\
\hline $\begin{array}{c}\text { Alvo } \\
\text { III }\end{array}$ & $\begin{array}{l}\text { Presidente } \\
\text { 1999: } 41.8 \% \\
\text { Taf 2000: } \\
58.2 \%\end{array}$ & $\begin{array}{l}\text { Passaredo } \\
\text { 1999: 84,4 \% } \\
\text { Varig 2000: } \\
15,6 \%\end{array}$ & $\begin{array}{l}\text { Passaredo } \\
\text { 1999: 78,3\% } \\
\text { Varig 2000: } \\
12,8 \%\end{array}$ & $\begin{array}{l}\text { Grupo Varig } \\
\text { 2000: } 62,2 \% \\
\text { Varig 2000: } \\
37,8 \%\end{array}$ & $\begin{array}{l}\text { Passaredo } \\
\text { 1999: 2,4\% } \\
\text { Presidente } \\
\text { 1999: } 77,6 \%\end{array}$ & $\begin{array}{l}\text { Varig 2000: } \\
17,1 \% \\
\text { Vasp 2000: } \\
59,8 \%\end{array}$ \\
\hline
\end{tabular}

Pesquisa Operacional, v.23, n.2, p.325-345, Maio a Agosto de 2003 


\begin{tabular}{|c|c|c|c|c|c|c|}
\hline & $\begin{array}{c}\text { Taf } \\
1999\end{array}$ & $\begin{array}{c}\text { Grupo } \\
\text { Transbrasil } \\
1999\end{array}$ & $\begin{array}{c}\text { Grupo } \\
\text { Transbrasil } \\
2000\end{array}$ & $\begin{array}{c}\text { Grupo Varig } \\
1999\end{array}$ & $\begin{array}{c}\text { Passaredo } \\
2000\end{array}$ & $\begin{array}{l}\text { Grupo Tam } \\
2000\end{array}$ \\
\hline & & & & & $\begin{array}{l}\text { Taf 2000: } \\
20 \%\end{array}$ & $\begin{array}{l}\text { Vasp 1999: } \\
23,1 \%\end{array}$ \\
\hline $\begin{array}{l}\text { Alvo } \\
\text { IV }\end{array}$ & $\begin{array}{l}\text { Taf 2000: } \\
100 \%\end{array}$ & $\begin{array}{l}\text { Vasp 2000: } \\
90,3 \% \\
\text { Vasp 1999: } \\
9,7 \%\end{array}$ & $\begin{array}{l}\text { Penta 1999: } \\
2,5 \% \\
\text { Vasp 2000: } \\
97,5 \%\end{array}$ & $\begin{array}{l}\text { Grupo Varig } \\
\text { 2000: } 84,3 \% \\
\text { Vasp 1999: } \\
15,7 \%\end{array}$ & $\begin{array}{l}\text { Presidente } \\
\text { 1999: } 35,2 \% \\
\text { Total 2000: } \\
64,8 \%\end{array}$ & - \\
\hline $\begin{array}{c}\text { Alvo } \\
\qquad V\end{array}$ & - & $\begin{array}{l}\text { Vasp 2000: } \\
100 \%\end{array}$ & $\begin{array}{l}\text { Passaredo } \\
\text { 1999: 8,7\% } \\
\text { Vasp 2000: } \\
91,3 \%\end{array}$ & $\begin{array}{l}\text { Varig 2000: } \\
100 \%\end{array}$ & $\begin{array}{l}\text { Passaredo } \\
\text { 1999: 2,4\% } \\
\text { Presidente } \\
\text { 1999: 97,6\% }\end{array}$ & - \\
\hline $\begin{array}{c}\text { Alvo } \\
\text { VI }\end{array}$ & - & $\begin{array}{l}\text { Passaredo } \\
\text { 1999: } 28,5 \% \\
\text { Varig 2000: } \\
14,2 \%\end{array}$ & $\begin{array}{l}\text { Penta 1999: } \\
15,1 \% \\
\text { Vasp 2000: } \\
68,2 \% \\
\text { Vasp 1999: } \\
16,7 \%\end{array}$ & $\begin{array}{l}\text { Varig 2000: } \\
98,4 \% \\
\text { Vasp 1999: } \\
1,6 \%\end{array}$ & $\begin{array}{l}\text { Presidente } \\
\text { 1999: 33,7\% } \\
\text { Trip 1999: } \\
66,3 \%\end{array}$ & - \\
\hline $\begin{array}{c}\text { Alvo } \\
\text { VII }\end{array}$ & - & $\begin{array}{l}\text { Penta 1999: } \\
28,5 \% \\
\text { Vasp 2000: } \\
23,9 \% \\
\text { Vasp 1999: } \\
47,6 \% \\
\end{array}$ & $\begin{array}{l}\text { Penta 1999: } \\
7,7 \% \\
\text { Vasp 2000: } \\
92,3 \%\end{array}$ & $\begin{array}{l}\text { Passaredo } \\
\text { 1999: 1,2\% } \\
\text { Varig 2000: } \\
98,8 \%\end{array}$ & $\begin{array}{l}\text { Presidente } \\
\text { 1999: 63,2\% } \\
\text { Total 2000: } \\
36,8 \%\end{array}$ & - \\
\hline $\begin{array}{r}\text { Alvo } \\
\text { VIII }\end{array}$ & - & $\begin{array}{l}\text { Passaredo } \\
\text { 1999: 8,3\% } \\
\text { Vasp 2000: } \\
91,7 \% \\
\end{array}$ & - & $\begin{array}{l}\text { Varig 2000: } \\
98,6 \% \\
\text { Vasp } 2000 \\
1,4 \%\end{array}$ & $\begin{array}{l}\text { Presidente } \\
\text { 1999: 61,9\% } \\
\text { Trip 1999: } \\
38,1 \%\end{array}$ & - \\
\hline $\begin{array}{l}\text { Alvo } \\
\text { IX }\end{array}$ & - & $\begin{array}{l}\text { Penta 1999: } \\
7,3 \% \\
\text { Vasp 2000: } \\
92,7 \%\end{array}$ & - & - & - & - \\
\hline
\end{tabular}

Os resultados para a Tam são de difícil análise, uma vez que o Grupo Tam passou por profundas transformações neste período, com a fusão e a eliminação de companhias internas do grupo, troca acentuada de frota e passagem gradual de companhia regional a internacional. Os principais alvos para esse grupo nos diversos anos são a Varig e a Vasp, o que retrata a situação intermediária de escala em que o grupo encontra-se atualmente.

A TRIP 2000 apresentava-se como eficiente segundo o modelo BCC, mas ela é de fato Pareto ineficiente dado que apresenta folga na variável passageiro.km oferecido. Assim, é necessário determinar benchmarks para esta DMU, que são apresentados na Tabela 6 . O principal bechmark é a TOTAL 2000. Deve-se considerar que esta companhia passou a operar apenas no setor cargueiro e de fretamentos, pelo que se pode concluir que a TRIP estava caminhando para operar numa faixa isolada, ou seja, com características diferentes de todas as outras companhias. 
Tabela 6 - Alvos alternativos para a companhias aérea TRIP em 2000.

\begin{tabular}{cc}
\hline \multirow{2}{*}{ Alvo I } & Penta 1999: 0,16267 \\
& Total 2000: 0,83733 \\
\hline \multirow{2}{*}{ Alvo II } & Penta 1999: 0,065089 \\
& Total 2000: 0,934911 \\
\hline
\end{tabular}

\section{Conclusões}

A separação em três modelos de análise de eficiência permitiu uma desagregação de variáveis, levando à descoberta de eficiências não evidentes e à explicação de algumas ineficiências. O principal caso de eficiência não esperada ocorreu no modelo de vendas com a companhia TAF. Esta companhia mostra-se ineficiente em modelos que consideram aspectos operacionais. Quando esses aspectos não foram considerados, a companhia apresenta eficiência crescente no tempo, o que sugere que sejam feitos estudos sobre sua estrutura de vendas e uso de frota.

O modelo operacional tem sua utilidade diminuída por negligenciar totalmente o mercado e por ter havido necessidade de restrições aos pesos, servindo apenas como um complemento de informações.

O modelo global reúne todas as informações, ainda necessitando de aprofundamentos na análise dos resultados, em especial na determinação de alvos para as DMUs ineficientes e no estudo da distribuição de pesos para as DMUs eficientes. Neste modelo não fica evidente o perfil da crise de 1999, que pode ser facilmente identificada no modelo de vendas. Comparando-se os modelos global e de vendas, pode-se sugerir que as companhias passaram a utilizar menos os recursos de que dispunham, em vez de diminuir esses recursos. Isto significa deixar aviões por mais tempo em terra e fazer menos uso de suas tripulações. Esta última prática é bastante corrente, uma vez que pessoal de vôo tem um salário fixo e um adicional por horas voadas. Estudos mais aprofundados, como por exemplo, uma análise da taxa de ocupação e tarifas praticadas, podem confirmar esta conclusão.

O uso do modelo DEA-Multiobjetivo permitiu a identificação de várias metas eficientes para as DMUs ineficientes. Isto possibilita aos gestores uma gama de opções gerenciais. Possibilita aos analistas descartar certas metas que são conhecidas na terminologia DEA como falsos positivos, ou seja, são eficientes apenas por certas particularidades matemáticas na relação entre seus inputs e outputs, e que podem não corresponder a uma eficiência real.

A aplicação do modelo é uma ferramenta de análise e diagnóstico, não de previsão. No entanto, uma análise acurada dos resultados pode fornecer indicações valiosas sobre tendências de comportamento futuros. Neste trabalho, os sérios problemas enfrentados atualmente pelo Grupo Transbrasil já eram detectáveis na análise dos alvos. É possível ainda verificar a possibilidade de sérios problemas para a companhia Passaredo, que foram infelizmente confirmados pela suspensão temporária das operações dessa companhia.

\section{Referências Bibliográficas}

(1) Adler, N. \& Golany, B. (2001). Evaluation of deregulated airline networks using data envelopment analysis combined with principal component analysis with an application to Western Europe. European Journal of Operational Research, 132(2), 260-273. 
(2) Allen, R.; Athassopoulos, A.; Dyson, R.G. \& Thanassoulis, E. (1997). Weight restrictions and value judgements in DEA: evolution, development and future directions. Annals of Operations Research, 73, 13-34.

(3) Angulo Meza, L. (2002). Um enfoque Multiobjetivo para determinação de alvos na Análise de Envoltória de Dados. Tese de Doutorado, PEP/UFRJ, Rio de Janeiro.

(4) Angulo Meza, L.; Gomes, E.G. \& Soares de Mello, J.C.C.B. (2002). Enfoque multiobjetivo para determinação de benchmarks de companhias aéreas brasileiras DEAineficientes. Anais do XVI ANPET, Natal, Outubro, 27-34.

(5) Angulo Meza, L. \& Lins, M.P.E. (2002). Modelos multiobjetivo para determinação de alvos na Análise Envoltória de Dados. Anais do XXXIV Simpósio Brasileiro de Pesquisa Operacional, Outubro, Rio de Janeiro.

(6) Angulo Meza, L. \& Lins, M.P.E. (2000). A Análise Envoltória de Dados através do uso do Frontier Analyst. Pesquisa Operacional, 19, 287-293.

(7) Banker, R.D.; Charnes, A. \& Cooper, W.W. (1984). Some models for estimating technical scale inefficiencies in Data Envelopment Analysis. Management Science, 30(9), 1078-1092.

(8) Burmann, P. (2000). Análise Prospectiva do Mercado de Carga Aérea no Brasil. Tese de Mestrado, COPPE/UFRJ, Rio de Janeiro.

(9) Charnes, A.; Cooper, W.W.; Lewin, A.Y. \& Seiford. L.M. (1995). Data Envelopment Analysis: Theory, Methodology and Applications. Kluwer Academic Publishers, USA.

(10) Charnes, A.; Cooper, W.W. \& Rhodes, E. (1978). Measuring the efficiency of decision-making units. European Journal of Operational Research, 2, 429-444.

(11) Charnes, A.; Gallegos, A. \& Li, H. (1996). Robustly efficient parametric frontiers via Multiplicative DEA for domestic and international operations of the Latin American airline industry. European Journal of Operational Research, 88(3), 525-536.

(12) Coelho, R. (2002). A construção da agenda de desregulamentação do setor de transporte aéreo no Brasil. In: Panorama Nacional da Pesquisa em Transportes 2002 - Anais do XVI ANPET [editado por J.R.A. Setti e E.N. Santos], Natal, Outubro, 2, 383-394.

(13) Cooper, W.W.; Seiford, L.M. \& Tone, K. (2000). Data Envelopment Analysis: A Comprehensive Text with Models, Applications, References and DEA-Solver Software. Kluwer Academic Publishers, USA.

(14) DAC (1992). Portaria 075/GM-5 de 06 de fevereiro de 1992. Departamento de Aviação Civil, Rio de Janeiro.

(15) DAC (1998). Portaria 701/DGAC de 30 de dezembro de 1998. Departamento de Aviação Civil, Rio de Janeiro.

(16) DAC (1999). Anuário de Dados Estatísticos - 1998. Departamento de Aviação Civil, Rio de Janeiro.

(17) DAC (2000). Anuário de Dados Estatísticos - 1999. Departamento de Aviação Civil, Rio de Janeiro. 
(18) DAC (2001) Anuário de Dados Estatísticos - 2000. Departamento de Aviação Civil, Rio de Janeiro.

(19) Espírito Santo Jr., R.A. (2000). Concentração no transporte aéreo e os possíveis impactos sobre os consumidores, a sociedade e a economia. Transporte em Transformação $V$, 155-170.

(20) Färe, R. \& Grosskopf, S. (1992). Malmquist productivity indexes and fisher ideal indexes. Economic Journal, 102, 158-160.

(21) Fernandes, E. \& Capobianco, H.M.P. (2000). Eficiência e estratégia financeira de empresas de aviação: uma comparação internacional. In: Análise Envoltória de Dados e perspectivas de integração no ambiente de Apoio à Decisão [editado por M.P.E. Lins e L. Angulo Meza], Editora da COPPE/UFRJ, Rio de Janeiro, 85-102.

(22) Fernandes, E. \& Pacheco, R.R. (2002). Efficient use of airport capacity. Transportation Research Part A: Policy and Practice, 36 (3), 225-238.

(23) Flap (2001). Aviação Comercial na América Latina. Flap Internacional, 349, 12-240.

(24) Gomes, E.G.; Soares de Mello, J.C.C.B.; Serapião, B.P.; Lins, M.P.E. \& Biondi, L.N. (2001). Avaliação de Eficiência de Companhias Aéreas Brasileiras: Uma Abordagem por Análise de Envoltória de Dados. (Eds.). In: Panorama Nacional da Pesquisa em Transportes 2001 - Anais do XV ANPET [editado por J.R.A. Setti e O.F. Lima Júnior], Campinas, Novembro, 2, 125-133.

(25) Lins, M.P.E. \& L. Angulo Meza (2000). Análise Envoltória de Dados e perspectivas de integração no ambiente de Apoio à Decisão. Editora da COPPE/UFRJ, Rio de Janeiro.

(26) Lucchesi, C. (2001). Regionais em busca do rumo certo. FLAP Internacional, 343, 26-44.

(27) Novaes, A.G.N. (2001a). Logística e Gerenciamento da Cadeia de Distribuição. Editora Campus, $1^{a}$ edição, Rio de Janeiro.

(28) Novaes, A.G.N. (2001b). Rapid-transit efficiency analysis with the Assurance-Region DEA method. Pesquisa Operacional, 21(2), 179-197.

(29) Portela, M.C.A.S.; Borges, P.C. \& Thanassoulis, E. (2002). Closest targets in nonoriented DEA models. Working Paper RP0205, Aston Business School, 34p.

(30) Serapião, B.P. (2001). Aplicação de Indicadores de Desempenho em Empresas Aéreas Utilizando o Gerenciamento de Receitas de Ativos Perecíveis. Tese de Mestrado, COPPE/UFRJ, Rio de Janeiro.

(31) Soares de Mello, J.C.C.B. (2002). Suavização da fronteira DEA com o uso de métodos variacionais. Tese de Doutorado, PEP/UFRJ, Rio de Janeiro.

(32) Soares de Mello, J.C.C.B.; Gomes, E.G.; Lins, M.P.E.; Assis, A.S. \& Biondi, L.N. (2002). Determinação de alvos em fronteiras DEA suavizadas. Anais do XXXIV Simpósio Brasileiro de Pesquisa Operacional, Outubro, Rio de Janeiro.

(33) Steuer, R.E. (1983). Operating Manual for the ADBASE Multiple Objective Linear Programming Package. Terry College of Business, University of Georgia, Athens, Georgia. 
Soares de Mello et al. - Análise de Envoltória de Dados no estudo da eficiência e dos benchmarks para companhias aéreas brasileiras

(34) Thanassoulis, E. \& Dyson, R.G. (1992). Estimating preferred target input-output levels using Data Envelopment Analysis. European Journal of Operational Research, 56, 80-97.

(35) Zhu, J. (1996). Data Envelopment Analysis with Preference Structure. Journal of the Operational Research Society, 47, 136-150. 